
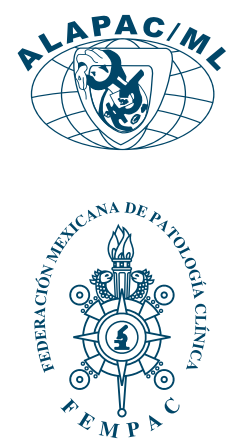

\title{
Reguladores de la expresión génica en cáncer de tiroides
}

\author{
Regulators of gene expression in thyroid cancer
}

Marrero Rodríguez María Teresa*

\author{
Palabras clave: \\ Microácido \\ ribonucleico, líneas \\ celulares, cáncer de \\ tiroides.
}

Keywords:

Micro ribonucleic acid, cell lines, thyroid cancer.

\footnotetext{
* Licenciada en Biología. Investigadora auxiliar. Instituto Nacional de Endocrinología. La Habana, Cuba.

\section{Correspondencia:}

Lic. María Teresa Marrero Rodríguez Instituto Nacional de Endocrinología Calle 29 y D. Plaza, CP 10400, La Habana, Cuba.

E-mail: mariat. marrero@infomed. sld.cu
}

\section{Recibido:} 02/07/2020 Aceptado: $10 / 09 / 2020$

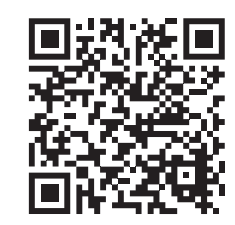

\section{RESUMEN}

El estudio de los mecanismos de regulación mediados por microácidos ribonucleicos ha arrojado resultados alentadores aplicables en el diagnóstico del cáncer. Objetivo: Estudiar el patrón de expresión de miR146b-5p y miR221-3p en líneas celulares de carcinoma de tiroides y su posible utilidad para el diagnóstico diferencial de estos tipos de tumores. Material y métodos: Se realizó un estudio de expresión de los microácidos ribonucleicos miR146b-5p y miR221-3p en líneas celulares de cáncer de tiroides BCPAD y $8505 \mathrm{c}$. Se realizaron ensayos de reacción en cadena de la polimerasa reverso transcriptasa (PCR-RT) y PCR reverso transcriptasa cuantitativa (PCR$R T q)$. Se utilizó el test $U$ de Mann-Whitney para comparar el nivel de expresión entre las líneas celulares $(p<0.05)$. Se encontraron diferencias significativas en la expresión de microácidos ribonucleicos entre las diferentes líneas celulares, el miR146b se expresó de forma significativa en la línea celular BCPAD, mientras que la expresión de miR221-3p fue significativamente mayor en la línea celular 8505. Conclusiones: Los perfiles de expresión de microácidos ribonucleicos estudiados se manifestaron de forma significativa en las líneas celulares de tumores tiroideos, por lo que pueden constituir nuevos biomarcadores para el diagnóstico del cáncer de tiroides.

\section{ABSTRACT}

The study of regulatory mechanisms mediated by micro ribonucleic acids has yielded encouraging results applicable in the diagnosis of cancer. Objective: To study the expression pattern of miR146b-5p and miR221-3p in thyroid carcinoma cell lines and its possible utility for the differential diagnosis of these types of tumors. Material and methods: An expression study of micro ribonucleic acids miR146b-5p and miR221-3p was performed in thyroid cancer cell lines $B C P A D$ and $8505 c$. Reverse transcriptase polymerase chain reaction $(P C R-R T)$ and quantitative reverse transcriptase PCR (PCR-RTq) assays were performed. The Mann-Whitney $U$ test was used to compare the level of expression between cell lines $(p<0.05)$. Significant differences were found in the expression of micro ribonucleic acids between the different cell lines, miR146b was significantly expressed in the BCPAD cell line, while the expression of miR221-3p was significantly higher in the 8505 cell line. Conclusions: Expression profiles of micro ribonucleic acids studied manifested themselves significantly in thyroid tumor cell lines, so they may constitute new biomarkers for the diagnosis of thyroid cancer.

\section{INTRODUCCIÓN}

U no de los avances recientes más sorprendentes en biología celular y molecular lo constituye la descripción de nuevos mecanismos de regulación de la expresión génica por parte de los microácidos ribonucleicos (miARN). ${ }^{1}$
Los miARN son estructuras moleculares de 20-22 nucleótidos con actividad postranscripcional que están implicados en la regulación de la expresión genética, se ha puesto de manifiesto su participación en distintas funciones fisiológicas y patológicas, tales como la apoptosis, la proliferación y la diferenciación celular, lo que demuestra su funcionalidad como genes

Citar como: Marrero RMT. Reguladores de la expresión génica en cáncer de tiroides. Rev Mex Patol Clin Med Lab. 2020 ; 67 (2): 85-88. doi: 10.35366/95552 
supresores de tumores o como protooncogenes en la carcinogénesis. ${ }^{1}$

Está ampliamente aceptado que la desregulación de los miARN juega un papel crítico en la carcinogénesis. El primer caso conocido de cáncer humano asociado con la desregulación de estas moléculas fue de leucemia linfocítica crónica. Más tarde, se encontró que muchos miARN tienen vínculos con algunos tipos de cáncer y de ahí que se les denomine «oncomirs». Algunos miARN se pueden comportar como genes supresores de tumores. ${ }^{2}$

El análisis del perfil de expresión de los miARN en neoplasias humanas ha revelado la presencia de perfiles de miARN para cada neoplasia, lo cual indica a los miARN como excelentes herramientas para el diagnóstico y pronóstico del cáncer. ${ }^{3}$

Se ha encontrado una serie de miARN implicada en cada una de las etapas de desarrollo del cáncer. Esto se debe posiblemente a dos factores: primero, que los miARN están regulados por oncogenes o genes supresores de tumor, ${ }^{4,5}$ por lo que son elementos importantes en el desarrollo tumoral; y en segundo lugar, se ha evidenciado que un gran número de miARN identificados en tejidos tumorales tiene dianas similares a los oncogenes o a los genes supresores de tumor, modulando las vías de expresión de estos genes, con lo que adquieren gran importancia en el entendimiento del desarrollo tumoral. ${ }^{6}$

Estudios recientes intentaron evaluar los perfiles de expresión de miARN específicos en el cáncer de tiroides, demostrando que la expresión persistente de miARN puede ser crucial para mantener la tumorogénesis. Sin embargo, aunque la expresión diferencial de moléculas de miARN diferentes ha sido reportada en cáncer de tiroides, los resultados en la literatura son inconsistentes. ${ }^{7,8}$

Tomando en consideración los aspectos antes expuestos, se propuso en este trabajo como objetivo estudiar el patrón de expresión de miR146b-5p y miR221-3p en líneas celulares de carcinoma de tiroides y su posible utilidad para el diagnóstico diferencial de estos tipos de tumores.

\section{MATERIAL Y MÉTODOS}

Se realizó un estudio de la expresión de miARN en líneas celulares de cáncer de tiroides.

Líneas celulares estudiadas:
- BCPAD: células de cáncer papilar de tiroides (CPT).

- 8505c: células de cáncer anaplásico de tiroides (CAT).

MicroARN estudiados:

- miR146b-5p y miR221-3p.

\section{Cultivo celular}

Para el cultivo de las líneas celulares se utilizó el medio de cultivo DMEM (medio de Eagle modificado por Dulbecco) suplementado con SFB (suero fetal bovino) al $10 \%$, penicilina/estreptomicina al $1 \%$, fungisona al $0.4 \%$ y $50 \mu \mathrm{g} / \mathrm{mL}$ de ácido ascórbico, con una atmósfera de $5 \%$ de $\mathrm{CO}_{2}$ a $37^{\circ} \mathrm{C}$.

\section{Cuantificación de ARN}

EI ARN total fue aislado de las células utilizando el reactivo TRIzol (Invitrogen, Carlsbad, CA, USA), siguiendo las instrucciones del proveedor. La concentración del ARN y su pureza se analizó midiendo la absorbancia a 260 y 280 nm en un espectrofotómetro (NanoDrop, Thermo Fisher Scientific Inc).

Se realizaron ensayos de reacción en cadena de la polimerasa reverso transcriptasa (PCR-RT) para la síntesis de ADN complementario (ADNc) de cadena simple de miARN y para la cuantificación la PCR reverso transcriptasa cuantitativa (PCR-RTq), para los cuales se utilizaron los NCode ${ }^{\mathrm{TM}}$ y los kits qRT-PCR (Invitrogen). La síntesis de $\mathrm{ADNc}$ se realizó a $37{ }^{\circ} \mathrm{C}$ durante 60 min, y la reacción se terminó por incubación a 95 ${ }^{\circ} \mathrm{C}$ durante 5 min.

La reacción de PCR-RTq incluyó un ciclo de 15 min a $95^{\circ} \mathrm{C}$ seguido de 40 ciclos, en el que cada ciclo consistió en 15 s a $94^{\circ} \mathrm{C}$, 30 s a $55^{\circ} \mathrm{C}$ y $70^{\circ} \mathrm{C}$ durante 30 s, y un ciclo de $95^{\circ} \mathrm{C}$ durante $1 \mathrm{~min}, 55^{\circ} \mathrm{C}$ durante 30 s y $95^{\circ} \mathrm{C}$ a $30 \mathrm{~s}$.

\section{Análisis estadístico}

Se utilizó la prueba de comparación de $U$ de MannWhitney para comparar la expresión de los microARN entre las líneas celulares. La diferencia fue considerada significativa para un valor de $p<0.05$.

Prueba $U$ de Mann-Whitney: prueba no paramétrica aplicada a dos muestras independientes. Utilizada para comparar dos grupos de rangos (medianas) y determinar 


\begin{tabular}{|c|c|c|c|c|}
\hline $\begin{array}{l}\text { Línea } \\
\text { celular }\end{array}$ & $\begin{array}{l}\text { miR146b-5p } \\
\text { (mediana) }\end{array}$ & $p$ & $\begin{array}{l}\text { miR221-3p } \\
\text { (mediana) }\end{array}$ & $p$ \\
\hline $8505 C$ & 0.59 & \multirow{2}{*}{0.0000} & 1.65 & \multirow{2}{*}{0.0046} \\
\hline BCPAD & 1.15 & & 0.82 & \\
\hline
\end{tabular}

que la diferencia no se deba al azar (que la diferencia sea estadísticamente significativa).

\section{RESULTADOS Y DISCUSIÓN}

En la literatura se plantea que la desregulación de miARN es un evento importante en la transformación de células tiroideas. ${ }^{9}$ Los perfiles de expresión de los miARN dependen del tipo de tumor, ${ }^{10}$ ya que la mayoría de ellos se expresan de forma tejido-específico y además difiere entre el tejido normal y el neoplásico. ${ }^{9}$

Estudios recientes han demostrado que la expresión de miARN en tumores de tiroides es importante para distinguir tumores malignos de benignos y que los diferentes histotipos de cáncer de tiroides muestran una expresión de miARN diferentes. ${ }^{11,12}$

Como se muestra en la Tabla 1 se encontró una sobreexpresión significativa del miR146b-5p en la línea celular BCPAD de CPT, mientras que en la línea celular 8505c de CAT fue significativa la expresión de los miR221-3p, resultados que coinciden con la literatura. El miR146b-5p ha sido descrito como el miARN expresado con mayor frecuencia en CPT, relacionando su mayor expresión con una mayor agresividad del tumor. ${ }^{13}$

El miR-146b está codificado en dos lugares diferentes, el 5q33 para el miR-146a y el 10q24 para el miR-146b. Ambos se encuentran sobreexpresados en el CPT, también en la variante folicular y en otros tumores no papilares. En la literatura se plantea que el miR146b-5p está sobreexpresado en CPT, distinguiendo de forma confiable este tipo de cáncer del carcinoma folicular (CFT) y de las lesiones benignas. ${ }^{11,14}$

Las familias miR-221 y miR-222 tienen una mayor expresión en tumores de peor pronóstico, como es cuando hay un mayor tamaño tumoral, invasión capsular, vascular o linfática o presencia de metástasis. ${ }^{15}$ En el Cancer Genome Atlas ${ }^{16}$ las familias de miR-221 y miR-222 se asociaron con tumores menos diferenciados como el CAT, éste se caracteriza por niveles elevados de miR-17 y miR-221/222, resultados simi- lares se obtuvieron en esta investigación en la que a pesar de que se encontró expresión en ambas líneas celulares estudiadas, fue significativamente mayor en la línea celular 8505 .

\section{CONCLUSIONES}

Los perfiles de expresión de microARN estudiados se manifestaron de forma significativa en las líneas celulares de tumores tiroideos, la expresión del miR146b-5p fue más significativa en el cáncer papilar del tiroides, mientras que en el cáncer anaplásico fue el miR221-3p, por lo que pueden constituir nuevos biomarcadores para el diagnóstico del cáncer de tiroides.

\section{REFERENCIAS}

1. Tang JT, Fang JY. MicroRNA regulatory network in human colorectal cancer. Mini Rev Med Chem. 2009; 9: 921-926.

2. Calin GA, Croce CM. MicroRNA signatures in human cancers. Nat Rev Cancer. 2006; 6: 857-866.

3. Volinia S, Calin GA, Liu CG, Ambs S, Cimmino A, Petrocca et al. A microRNA expression signature of human solid tumors defines cancer gene targets. Proc Natl Acad Sci. 2006; 103: 2257-2261.

4. Kosaka N, Iguchi $\mathrm{H}$, Ochiya T. Circulating mi-croRNA in body fluid: a new potential biomarker for cancer diagnosis and prognosis. Cancer Sci. 2010; 101 (10): 2087-2092.

5. Roth C, Rack B, Müller V, Janni W, Pantel K, Schwar-Zenbach H. Circulating microRNAs as blood-based markers for patients with primary and metastatic breast cancer. Breast Cancer Res. 2010; 12 (6): 1186-2766.

6. Allegra A, Alonci A, Campo S, Penna G, Petrungaro A, Gerace D et al. Circulating microRNAs: new bio-markers in diagnosis, prognosis and treatment of cancer. Int J Oncol. 2012; 41 (6): 1897-1912.

7. Yu S, Liu Y, Wang J et al. Circulating MicroRNA profiles as potential biomarkers for diagnosis of papillary thyroid carcinoma. J Clin Endocrinol Metab. 2012; 97: 2084-2092.

8. Fuziwara CS, Kimura ET. MicroRNA deregulation in anaplastic thyroid cancer biology. Int J Endocrinol. 2014; 2014: 743450.

9. Nikiforova MN, Tseng GC, Steward D, Diorio D, Nikiforov YE. MicroRNA expression profiling of thyroid tumors: biological significance and diagnostic utility. J Clin Endocrinol Metab. 2008; 93 (5): 1600-1608.

10. Marini F, Luzi E, Brandi ML. MicroRNA role in thyroid cancer development. J Thyroid Res. 2011; 2011: 407123.

11. Sheu SY, Grabellus F, Schwertheim S, Worm K, Broecker-Preuss M, Schmid KW. Differential miARN expression profiles in variants of papillary thyroid carcinoma and encapsulated follicular thyroid tumours. Br J Cancer. 2010; 102 (2): 376-382.

12. Shen R, Liyanarachchi S, Li W, Wakely PE Jr, Saji M, Huang J et al. MicroRNA signature in thyroid fine needle aspiration cytology applied to "atypia of undetermined significance" cases. Thyroid. 2012; 22 (1): 9-16.

13. Pallante P, Battista S, Pierantoni GM, Fusco A. Deregulation of microRNA expression in thyroid neoplasias. Nat Rev Endocrinol. 2014; 10 (2): 88-101.

14. Chen YT, Kitabayashi N, Zhou XK, Fahey TJ 3rd, Scognamiglio T. MicroRNA analysis as a potential diagnostic tool for papillary thyroid carcinoma. Mod Pathol. 2008; 21 (9): 1139-1146. 
15. Wójcicka A, Kolanowska M, Jażdżewski K. Mechanisms in endocrinology: MicroRNA in diagnostics and therapy of thyroid cancer. Eur J Endocrinol. 2016; 174 (3): R89-R98.
16. Cancer Genome Atlas Research Network. Integrated genomic characterization of papillary thyroid carcinoma. Cell. 2014; 159 (3): 676-690.

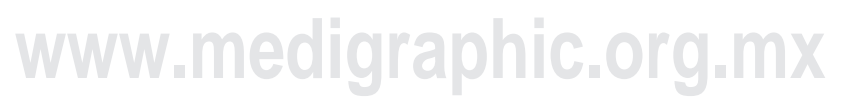

